\title{
Dynamic Dominating Set and Turbo-Charging Greedy Heuristics
}

\author{
Rodney G. Downey, Judith Egan, Michael R. Fellows, \\ Frances A. Rosamond*, and Peter Shaw
}

\begin{abstract}
The main purpose of this paper is to exposit two very different, but very general, motivational schemes in the art of parameterization and a concrete example connecting them. We introduce a dynamic version of the DOMINATING SET problem and prove that it is fixed-parameter tractable (FPT). The problem is motivated by settings where problem instances evolve. It also arises in the quest to improve a natural greedy heuristic for the DOMINATING SET problem.
\end{abstract}

Key words: kernelization; multivariate algorithms; parameterized algorithms; turbo-charging; heuristics

\section{Introduction}

Suppose we have a network (modeled by an undirected graph) $G$, and we have a dominating set $D$ for $G$. We do not assume that $D$ is optimal, but we do assume that we are happy with $D$. Or perhaps we are stuck with $D$ because we have built facilities on the vertices of $D$. Unfortunately, as often happens, $G$ changes to $G^{\prime}$ and $D$ is no longer a dominating set. Fortunately the changes were relatively minor: A few edges disappeared and a few were added.

We might reasonably hope that if we can find a way to slightly modify $D$ to obtain a set of vertices $D^{\prime}$ that is a dominating set of $G^{\prime}$, then we will once again be happy, or at least functional.

The above discussion leads to the following problem. Here, $d_{\mathrm{e}}\left(G, G^{\prime}\right)$ denotes the edge edit distance from $G$ to $G^{\prime}$ : the number of edge edit operations (edge

- Rodney G. Downey is with School of Mathematics, Statistics and Operations Research, Victoria University of Wellington, Wellington 600, New Zealand. E-mail: Rod.Downey@msor.vuw.ac.nz.

- Judith Egan, Michael R. Fellows, Frances A. Rosamond, and Peter Shaw are with School of Engineering and Information Technology, Charles Darwin University, Darwin, NT 0909, Australia. E-mail: Judith. Egan@cdu.edu.au; Michael.Fellows@cdu.edu.au; Frances. Rosamond@cdu.edu.au; Peter.Shaw@cdu.edu.au.

* To whom correspondence should be addressed. Manuscript received: 2014-06-20; accepted: 2014-06-27 addition or edge deletion) required to transform $G$ into $G^{\prime}$. Equivalently, $d_{\mathrm{e}}\left(G, G^{\prime}\right)=\mid\left(E(G)-E\left(G^{\prime}\right)\right) \cup$ $\left(E\left(G^{\prime}\right)-E(G)\right) \mid$. Similarly, the vertex edit distance from $D$ to $D^{\prime}$ is denoted $d_{\mathrm{v}}\left(D, D^{\prime}\right)=\mid\left(D-D^{\prime}\right) \cup$ $\left(D^{\prime}-D\right) \mid$. See Fig. 1 .

\section{DYNAMic DOMINATING SET (DDS)}

Instance: A graph $G$ and a graph $G^{\prime}$ with $d_{\mathrm{e}}\left(G, G^{\prime}\right) \leqslant k$; a dominating set $D \subseteq V(G)$; a positive integer $r$.

Parameter: $(k, r)$.

Question: Does there exist a set of vertices $D^{\prime} \subseteq V(G)=V\left(G^{\prime}\right)$ with $d_{\mathrm{v}}\left(D, D^{\prime}\right) \leqslant r$ and with $D^{\prime}$ a dominating set of $G^{\prime}$ ?

Another motivation. The above storyline is quite natural and could be widely relevant. Essentially,

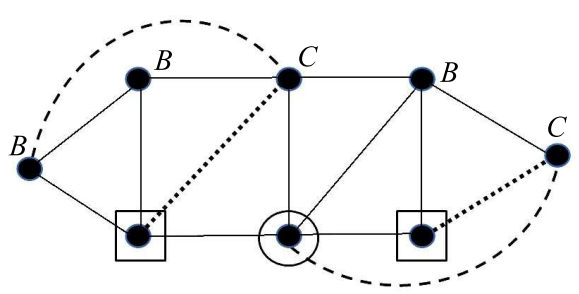

Fig. 1 The initial graph $G$ consisted of the edges indicted by and .... The new graph $G^{\prime}$ consists of the edges indicated by _ _ and . . .. In other words, ...... indicates edge delection and - - - indicates edge addition in the passage from $G$ to $G^{\prime}$. In this example $d_{\mathrm{e}}\left(G, G^{\prime}\right)=4$. The two vertices marked with a square are a dominating set $D$ in $G$. These, together with the vertex marked with a circle form a dominating set $D^{\prime}$ of $G^{\prime}$. 
we have an instance $I$ and solution $S$ with which we are content, or at least functional. Instance $I$ evolves (slightly) into $I^{\prime}$. If we can slightly modify $S$ into $S^{\prime}$, then we will again be content, or at least functional. This is a strong storyline in the context of practical computing.

There is another, completely different but also quite general, motivation to our problem. Most work in algorithmics and complexity to date could be characterized as top down. One models the entire computational problem, hoping to find a tractability result that performs well on real-world datasets. This approach usually fails, and most practical computing relies on heuristics, as recently emphasized by Karp ${ }^{[1]}$.

The multivariate approach to algorithmics pioneered in the important work of Chen et al. ${ }^{[2-11]}$ and others $^{[12-15]}$, offers the framework for a plausible alternative. This alternative is to work bottom up: begin with a successful heuristic $H$ used in practice, and try to find a fixed-parameter tractable (FPT) subroutine to empower a sharper heuristic $H^{\prime}$. A possible strength of this approach is that successful heuristics capture some implicit knowledge of real-world datasets, a matter of crucial importance, but also significant mystery. We do not understand natural datasets very well. As emphasized by Karp ${ }^{[1]}$ in recent years, we generally do not understand why heuristics work so well in practice for NP-hard problems. In the bottom-up approach, we begin with a successful heuristic, and try to improve it.

Greedy heuristics (and greedy subroutines) play a major role in practical computing. In the quest to deploy FPT subroutines to improve greedy heuristics there arises a very general scheme of parameterization.

Consider the following greedy algorithm for Minimum Dominating SeT:

(1) List the vertices of $G$ from high degree to low degree.

(2) Going through the list, if a vertex $v$ is not dominated by the vertices already chosen to belong to the dominating set $D$, choose a vertex in $N[v]$ (the vertex $v$ together with all of its neighbors) of highest utility (the number of sofar undominated neighbors).

As this greedy algorithm is executed on a graph $G$, one may suffer a moment of regret: At some point (at a vertex $v$ on the list) one may be forced to add another vertex to $D$ from $N[v]$. The regret comes from the fact that our objective is to minimize the size of the resulting dominating set $D$.

Notice that this greedy algorithm is somewhat nondeterministic (as is often the case): When one is forced to add a new vertex to $D$, there might be several possible choices.

The key idea in the following parameterized problem, in view of the nondeterminism of the greedy algorithm, is to back up, with the distance of the back-up being the parameter $k$, and to ask if there is a different route forward through the list, redoing the last $k$ steps, that avoids the moment of regret. That is, the route should reach $v$ with a current dominating set $D^{\prime}$ with $\left|D^{\prime}\right|<|D|$, where $D$ is the current dominating set that we would have by adding a new vertex to $D$ when we reach $v$. Possibly, this may lead to an improved greedy heuristic. Formalizing this discussion, we have the following problem.

\section{GREEDY IMPROVEMENT FOR DOMINATING SET (GREEDY DS)}

Instance: A graph $G$; a list $L$ of the vertices of $G$ ordered from highest degree to lowest degree, $L=\left(v_{1}, \cdots, v_{l}, \cdots, v_{l+k}=\right.$ $\left.v, \cdots, v_{n}\right)$ ( $v$ is the moment of regret, $v_{l}$ is the back-up point, and $k$ is the amount of back-up); a set of vertices $D \subseteq V(G)$ that dominates the set $V^{\prime}$ of vertices in $G$, $V^{\prime}=\left\{v_{1}, \cdots, v_{l+k}=v\right\}$ (note that $D$ is not necessarily a subset of $V^{\prime}$ ); a partition $D=D_{1} \cup D_{2}$ where $D_{1}$ dominates the set of vertices $\left\{v_{1}, \cdots, v_{l}\right\}$ and $\left|D_{2}\right| \leqslant k$.

Parameter: $k$.

Question: Is there a set of vertices $D^{\prime} \subseteq$ $V(G)$ such that $D^{\prime}$ dominates the vertices of $V^{\prime} \cup D$, with $D_{1} \subseteq D^{\prime}$ and $\left|D^{\prime}\right|<|D|$ ?

\subsection{Our results}

We offer two main results.

Theorem 1 Dynamic Dominating SET is fixedparameter tractable.

Theorem 2 GREEDY IMPROVEMENT FOR Dominating SET is fixed-parameter tractable.

Although the two motivations are quite different, and are both of wide relevance in the context of heuristics and practical computing situations for NPhard problems, it turns out that the two problems are closely related.

We also offer some results of secondary importance, but still of interest. 
Theorem 3 Dynamic Dominating Set is NPhard. The motivation to this result is that FPT results should generally be a response to NPcompleteness. Said differently, the best kind of FPT is solvability in polynomial time!

Theorem 4 Although Dynamic Dominating SET is fixed-parameter tractable, it does not admit a polynomial kernel unless NP $\subseteq$ co-NP/poly.

\subsection{Related work}

Dynamic Dominating Set (and the entire program that it represents) is closely related to the body of work on re-optimization ${ }^{[16,17]}$.

The difference between the re-optimization problem template and the dynamic problem template, is that in the re-optimization research program one assumes that for the original instance $I$, the solution $S$ is optimal, and one tries to restore this situation for $S^{\prime}$ with respect to $I^{\prime}$. In the classical complexity setting, most reoptimization problems turn out to be NP-hard. In the re-optimization setting, it is natural to parameterize on $(k, r)$, just as we have in the definition of DYNAMIC DOMINATING SET.

We do not prove this here, but the naturally defined Re-optimization AnAlog of Dynamic DOMINATING SET is $W[2]$-complete (the proof is not difficult), and as in the classical framework, most parameterized complexity results in the re-optimization program are hardness results.

In rough terms, optimality seems to be the "kiss of death", and everything becomes hard for such variations in problem definition. The following seemingly natural variation is also $W[2]$-complete, as first noted by Stege ${ }^{[18]}$ (private communication).

\section{Almost A Dominating Set \\ Instance: A graph $G$ and $D \subseteq V(G)$; a positive integer $k$.}

Parameter: $k$.

Question: Does there exist a set $D^{\prime} \subseteq$ $V(G)$, with $d_{v}\left(D, D^{\prime}\right) \leqslant k$, where $D^{\prime}$ is a dominating set in $G$ ?

There have been a number of papers investigating the complexity of "dynamic problems" in various application domains ${ }^{[19-21]}$, but only one (to the best of our knowledge) that investigates the parameterized complexity of an dynamic problem - the recent pioneering paper of Hartung and Niedermeier ${ }^{[22]}$ that inspired this paper.

\section{Preliminary Definitions and Background}

A parameterized problem is classified FPT if it can be solved by an algorithm with running time $f(k) \cdot n^{O(1)}$, where $f$ is some arbitrary function depending only on the parameter $k$, not on the input size $n^{[12,15]}$. A parameterized reduction from a parameterized problem $\Pi$ to a parameterized problem $\Pi^{\prime}$ is an algorithm that, given an instance $(I, k)$ of $\Pi$, computes in FPT time an instance $\left(I^{\prime}, k^{\prime}\right)$ of $\Pi^{\prime}$, with $k^{\prime}$ only depending on $k$, such that $(I, k)$ is a yes-instance of $\Pi$ if and only if $\left(I^{\prime}, k^{\prime}\right)$ is a yes-instance of $\Pi^{\prime}$. A classification framework for the complexity analysis of parameterized problems is supplied by the $W$-hierarchy introduced by Downey and Fellows ${ }^{[12]}$.

A kernelization of a parameterized problem $\Pi$ is a polynomial-time algorithm that, given an instance $(I, k)$ of $\Pi$, computes an instance $\left(I^{\prime}, k^{\prime}\right)$ of $\Pi$, where

(1) $\left|I^{\prime}\right| \leqslant f(k)$ for some function $f$,

(2) $k^{\prime} \leqslant g(k)$ for some function $f$, and

(3) $(I, k)$ is a yes-instance of $\Pi$ if and only if $\left(I^{\prime}, k^{\prime}\right)$ is a yes-instance of $\Pi$.

Importantly, it is known (and not hard to show) that a parameterized problem is FPT if and only if it admits a kernelization $^{[4,23]}$.

A kernelization algorithm is often based on a set of data-reduction rules that can be applied in polynomial time, to progessively simplify $(I, k)$ to the reduced instance $\left(I^{\prime}, k^{\prime}\right)$. The kernel of the problem instance $(I, k)$ is the reduced instance $\left(I^{\prime}, k^{\prime}\right)$ and the size of the kernel is given by $\left|I^{\prime}\right|$. If the kernel size is guaranteed to be bounded by a polynomial function of $k$ then the problem is said to have a polynomial kernel. In Section 4 we will employ some recently developed techniques of Refs. [24-26] for demonstrating that an FPT parameterized problem has no polynomial kernel.

Introductory and further information about parameterized complexity is presented in Refs. [12-15, 27].

Given a graph $G=(V, E)$, let $V(G)=V$ and $E(G)=E$. Unless otherwise stated, we will assume that all graphs are finite, simple, and undirected. Two vertices $u$ and $v$ in $V(G)$ are neighbors, if $(u, v)$ is an edge in $E(G)$. The set of neighbors, or open neighborhood, of a vertex $v$ is given by $N_{G}(v)=$ $\{u \in V(G) \mid(u, v) \in E(G)\}$. The closed neighborhood of $v$ is $N_{G}[v]=N_{G}(v) \cup\{v\}$. If $S \subseteq V(G)$, then $N_{G}(S)=\bigcup_{v \in S} N_{G}(v)$ and $N_{G}[S]=N_{G}(S) \cup S$. A dominating set for $G$ is a set of vertices $S \subseteq V(G)$ such 
that every vertex not in $S$ has at least one neighbor in $S$. Equivalently, $S$ is a dominating set for $G$ if $N_{G}[S]=$ $V(G)$.

If $C$ and $D$ are subsets of $V(G)$ satisfying $C \subseteq$ $N_{G}[D]$, then we say that $D$ dominates $C$, or equivalently, that $C$ is dominated by $D$. Note that $D$ may also dominate vertices outside of $C$ but it need not be a dominating set for $G$. When a single vertex $\{v\}$ is dominated by $D$, we say that $v$ is dominated by $D$. Otherwise, $N_{G}[v] \cap D=\varnothing$, hence $v$ is called a non-dominated vertex, with respect to $D$.

A set of vertices $J \subseteq V(G)$ is an independent set if $N_{G}(J) \cap J=\varnothing$. A set of vertices $C \subseteq V(G)$ is called a vertex cover of $G$ if every edge of $G$ is incident with a vertex in $C$. That is, $C=\{u \in V(G) \mid \forall(v, w) \in$ $E(G), u=v$ or $u=w\}$.

The Dominating SeT problem is well-known to be NP-complete ${ }^{[28]}$.

\section{DOMINATING SET}

Input: A graph $G=(V, E)$ and a positive integer $k$.

Question: Does there exist $D \subseteq V$ such that $|D| \leqslant k$, and $D$ is a dominating set for $G$ ?

Parameterized by $k$ (the size of the dominating set), Dominating $\operatorname{SeT}(k)$ is $W[2]$-complete ${ }^{[12]}$.

Given two graphs possessing the same set of vertices, that is $G=(V, E)$ and $G^{\prime}=\left(V, E^{\prime}\right)$, we interpret that $G^{\prime}$ has been obtained from $G$ by some sequence of edge edit operations where the allowable such operations are edge deletion and edge addition. We define the edge edit distance, $d_{\mathrm{e}}\left(G, G^{\prime}\right)$ as the Hamming distance between $0 / 1$ vectors indicating the edge sets, $E$ and $E^{\prime}$, of the two graphs. In this paper, we are concerned with the situation where we have some solution $D \subseteq$ $V$ for $G$ and are interested in whether or not there exists a solution $D^{\prime} \subseteq V$ for the modified graph $G^{\prime}$, such that $D^{\prime}$ is within a certain distance of the original solution. This distance is the vertex solution set distance, $d_{\mathrm{v}}\left(D, D^{\prime}\right)$ which is defined to be the Hamming distance between $0 / 1$ vectors indicating the sets of vertices, $D, D^{\prime}$, of solutions corresponding to $G, G^{\prime}$, respectively (the vertex edit distance between the two sets of vertices).

\section{Dynamic Dominating Set is FPT}

We first show that Dynamic Dominating Set is polynomial-time equivalent (with a nice connection between the parameters) to the following problem with a simplified structure.

\section{Monotone Dynamic Dominating Set (MON-DDS)}

Instance: A graph $G$; a graph $G^{\prime}$ with $V(G)=V\left(G^{\prime}\right)$ and $d_{\mathrm{e}}\left(G, G^{\prime}\right) \leqslant k$ where $G^{\prime}$ is obtained from $G$ by only the deletion of edges (thus $E\left(G^{\prime}\right) \subseteq E(G)$ ); a dominating set $D \subseteq V(G)$ of $G$; a positive integer $r$.

Parameter: $(k, r)$.

Question: Does there exist a set of vertices $D^{\prime} \subseteq V(G)=V\left(G^{\prime}\right)$ with $d_{\mathrm{v}}\left(D, D^{\prime}\right) \leqslant r$ where $D^{\prime}$ is obtained from $D$ only by adding vertices (thus $D \subseteq D^{\prime}$ ), and with $D^{\prime}$ a dominating set of $G^{\prime}$ ?

Lemma 1 Dynamic Dominating Set and Monotone Dynamic Dominating SET are polynomial-time equivalent.

Proof We must argue that there are reductions in both directions between the two problems.

We first reduce MONOTONE DYNAMIC Dominating SET to DYNAMIC Dominating SET. Suppose $(I,(k, r))$ is an instance of Mon-DDS where $I$ describes $G, G^{\prime}$, and $D$. We transform by simply taking $(I,(k, r))$ to be the input to DDS (the identity transformation).

If $(I,(k, r))$ is a yes-instance of MON-DDS, as witnessed by $D^{\prime}$, then $D^{\prime}$ also witnesses that $(I,(k, r))$ is a yes-instance of DDS.

Conversely, suppose a set of vertices $D^{\prime}$ witnesses that $(I,(k, r))$ is a yes-instance of DDS. Then $D^{\prime}$ is obtained from $D$ by a sequence $\sigma$ of at most $r$ vertex edit operations. Consider $D^{\prime \prime}$ to be the vertex set obtained by restricting $\sigma$ to only the vertex addition operations. Clearly we have $d_{\mathrm{v}}\left(D, D^{\prime}\right) \leqslant r$. Since $D \subseteq D^{\prime \prime}$ and $D$ is a dominating set of $G, D^{\prime \prime}$ is also a dominating set of $G$. Since $G^{\prime}$ is obtained from $G$ only by adding edges, $D^{\prime \prime}$ is also a dominating set for $G^{\prime}$, and thus $D^{\prime \prime}$ witnesses that $(I,(k, r))$ is a yes-instance for MON-DDS.

Reducing in the other direction, suppose $(I,(k, r))$ is an instance of DDS where $I$ describes $G, G^{\prime}$, and $D$. We transform as follows to an instance $\left(I^{\prime},(k, r)\right)$ of MON-DDS where $I^{\prime}$ is composed of information about a graph $G^{+}$and the graph $G^{\prime}$ where $G^{\prime}$ is obtained from $G^{+}$only by deleting edges. We know that $G^{\prime}$ is obtained from $G$ by a sequence $\sigma$ of at most $k$ operations of edge 
addition and edge deletion. $G^{+}$is the graph we obtain from $G$ by restricting $\sigma$ to only the operations of edge addition. This, $G^{\prime}$ is obtained from $G^{+}$by only edge deletion operations, as required for a valid instance of Mon-DDS; also $d_{\mathrm{e}}\left(G^{+}, G^{\prime}\right) \leqslant k$.

If $(I,(k, r))$ is a yes-instance of DDS, as witnessed by a set of vertices $D^{\prime}$, so that $D^{\prime}$ is obtained from $D$ by at most $r$ vertex edit operations, let $D^{\prime \prime}$ denote the set of vertices one obtains by restricting to only the vertex additions. So $d_{\mathrm{v}}\left(D, D^{\prime \prime}\right) \leqslant r$ and also $D^{\prime} \subseteq D^{\prime \prime}$, so $D^{\prime \prime}$ is also a dominating set for $G^{\prime}$ and thus witnesses that $\left(I^{\prime},(k, r)\right)$ is a yes-instance of DDS.

Conversely, suppose a set of vertices $D^{\prime}$ is a witness that $\left(I^{\prime},(k, r)\right)$ is a yes-instance of MoN-DDS, but then $D^{\prime}$ is also a witness that $(I,(k, r))$ is a yes-instance of DDS.

The above lemma shows that DDS and MoNDDS are equivalent with respect to all measures of computational complexity considered in this paper.

Our proof that DDS (equivalently, MoN-DDS) is FPT will involve a reduction to the following problem of possible independent interest:

\section{Dominating A Vertex Cover (Dom-A- $\mathrm{VC})$}

Input: A graph $G=(V, E)$, a vertex cover $C$ for $G$ of size $k$, and a positive integer $r \leqslant k$.

Parameter: $(k, r)$.

Question: Does there exist $D \subseteq V$ such that $|D| \leqslant r$, and $D$ dominates the vertex cover $C$ ?

Lemma 2 An instance $(I,(k, r))$ of the problem MON-DDS can be reduced in polynomial time to an instance $\left(I^{\prime},(k, r)\right)$ of DoM-A-VC.

Proof Let $(I,(k, r))$ where $I$ gives us information about $G, G^{\prime}$, and $D$, be an instance of Mon-DDS. Let $C=V\left(G^{\prime}\right) \backslash N_{G^{\prime}}[D]$, and $B=N_{G^{\prime}}(D) \backslash D$. The sets $C, B$, and $D$ form a partition of the vertices of $G^{\prime}$. Since at most $k$ edge addition operations transformed $G$ to $G^{\prime}$, the set $C$ consists of at most $k$ non-dominated vertices. If $k \leqslant r$ then we are done, take $D^{\prime}=D \cup C$. Henceforth, assume that $k>r$.

Considering that vertices in $C$ can only be dominated by the vertices in $B$ or $C$ in augmenting $D$ to $D^{\prime}$ proves the soundness of the reduction rules:

R1: If $v \in D$, then remove $v$ and its incident edges.
R2: If $v \in B$ and $N(v) \cap C=\varnothing$, then remove $v$ and its incident edges.

R3: If $(u, v)$ is an edge and $\{u, v\} \subseteq B$, remove the edge $(u, v)$.

Thus, we obtain from $G^{\prime}$ a reduced graph $H$ such that,

$$
\begin{aligned}
& V(H)=V\left(G^{\prime}\right) \backslash X \text { and } E(H)=E\left(G^{\prime}\right) \backslash Y, \text { where, } \\
& X=D \cup\left\{v \in B \mid N_{G^{\prime}}(v) \cap C=\varnothing\right\}, \text { and } \\
& Y=\{(u, v) \mid\{u, v\} \cap X \neq \varnothing\} \cup\{(u, v) \mid\{u, v\} \subseteq B\} .
\end{aligned}
$$

In $H$, what remains of $B$ now forms an independent set, so it means $C$ is a vertex cover for $H$. The original instance $I$ of $\operatorname{MoN}-\operatorname{DDS}(k, r)$ is thus reduced to an instance $I^{\prime}=(H, C, k, r)$ of the problem DoM-A$\operatorname{VC}(k, r)$ where $C$ is a vertex cover for $H,|C| \leqslant k$. As required, the reduction can be achieved in polynomial time.

If $I^{\prime}$ is a yes-instance, then $H$ has a set of vertices $D$, of size at most $r$, and such that $D$ dominates $C$. Then in $I$, the graph $G^{\prime}$ has a dominating set $D^{\prime}=D \cup C$ with $d_{\mathrm{v}}\left(D, D^{\prime}\right) \leqslant r$, hence $I$ is a yes-instance. Conversely, suppose that $I$ is a yes-instance so that $G^{\prime}$ has a dominating set $D^{\prime}$ with $d_{\mathrm{v}}\left(D, D^{\prime}\right) \leqslant r$. Then in $I^{\prime}$, the graph $H$ has a set of vertices $D=D^{\prime} \backslash D$, hence $|D| \leqslant r$, and $D$ dominates the vertices in $C$. Thus $I^{\prime}$ is a yes-instance. Hence the lemma is shown.

Lemma 3 The problem Dom-A-VC $(k, r)$ is FPT.

Proof Given an instance $(G, C, k, r)$ of DoM-A$\operatorname{VC}(k, r)$, let $B=V(G) \backslash C$. Since $C$ is a vertex cover, the vertices in $B$ form an independent set. The question is whether or not there is a set $D \subset V(G),|D| \leqslant r$, such that $D$ dominates $C$. If $k \leqslant r$ the solution is trivial, take $D=C$. Thus $k>r$.

Two vertices $u, v \in B$ are said to have the same neighbor type if $N_{G}(u)=N_{G}(v)$. If there are multiple vertices in $B$ of the same neighbor type then we can discard all but one of them to dominate that neighborhood. The size of the vertex cover, $|C|=k$, so there are $2^{k}$ possible neighbor types to consider in $B$. Some of the vertices in $D$ may come from $C$ so we also add those for consideration. Thus we need to search at most $\left(\begin{array}{c}k+2^{k} \\ r\end{array}\right)$ possibilities.

We now state our first main result in Theorem 5 (called Theorem 1 in the Introduction).

Theorem 5 The problem DDS is FPT.

Proof Immediate from Lemmas 1-3 and the equivalence of DDS and Mon-DDS. 


\section{A Lower Bound on the Kernel Size}

The proof of Lemma 3 identified a kernel for DDS $(k, r)$ that is exponential in $k$. As will be shown in this section, a polynomial kernel for the problem is unlikely.

Techniques introduced by Bodlaender et al. ${ }^{[24]}$, and Bodlaender et al. ${ }^{[25]}$, together with a result by Fortnow and Santhanam ${ }^{[26]}$, provide a framework for many problems to be classified as having no polynomial kernel unless NP $\subseteq$ coNP / Poly. Successful applications of that framework includes the work of Dom et al. ${ }^{[29]}$ who showed that a list of parameterized problems, including RED-Blue Dominating SET which we will soon consider, have no polynomial kernel (unless NP $\subseteq$ coNP/Poly).

A concept used by Binkel-Raible et al. ${ }^{[30]}$ and formalised in Bodlaender et al. ${ }^{[25]}$ is that of a Polynomial Parameter and Time (PPT) reduction. A PPT reduction is a parameterized reduction from a parameterized problem $\Pi$ to a parameterized problem $\Pi^{\prime}$, taking an instance $(I, k)$ to an instance $\left(I^{\prime}, k^{\prime}\right)$ in time polynomial in $|(I, k)|$, with the additional constraint that $k^{\prime}$ is bounded by $k^{c}$, for some constant $c$. All of the reductions so far discussed in this paper can be seen to be PPT reductions.

Theorem 6 is given by Bodlaender et al. ${ }^{[25]}$

Theorem 6 Let $P$ and $Q$ be parameterized problems. Suppose that the unparameterized versions of these problems are, respectively, $\widetilde{P}$ and $\widetilde{Q}$, and furthermore that $\widetilde{P}$ is NP-complete and $\widetilde{Q}$ is in NP. If $Q$ has a polynomial kernel and there is a PPT reduction from $P$ to $Q$, then $P$ has a polynomial kernel.

The contrapositive of Theorem 6 is stated next as a corollary of Theorem 6 .

Corollary 1 Let $P$ and $Q$ be parameterized problems. Suppose that the unparameterized versions of these problems are, respectively, $\widetilde{P}$ and $\widetilde{Q}$, and furthermore that $\widetilde{P}$ is NP-complete and $\widetilde{Q}$ is in NP. If $P$ has no polynomial kernel (unless NP $\subseteq$ coNP / Poly) and there is a PPT reduction from $P$ to $Q$, then $Q$ has no polynomial kernel unless NP $\subseteq$ coNP / Poly.

For a more thorough explanation of kernelization lower bounds techniques, including the results mentioned herein, we refer the interested reader to

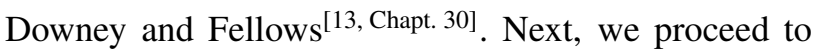
apply Corollary 1 to show that DDS has no polynomial kernel unless NP $\subseteq$ coNP / Poly.

Lemma 4 The unparameterized version of DDS is NP-complete.
Proof Observe that DoM-A-VC is in NP.

Let $I=(H, d)$ be an instance of Dominating SET and transform $I$ as follows. Let $G$ be the graph obtained from $H$ by first adjoining a single vertex $u^{*}$ and then adding an edge $\left(u^{*}, v\right)$ to every other vertex $v$ in $G$. Specifically, we have $V(G)=V(H) \cup\left\{u^{*}\right\}$, and $E(G)=E(H) \cup E^{*}$ where $E^{*}=\left\{\left(u^{*}, v\right) \mid v \in V(G), v \neq u^{*}\right\}$. Let $D=$ $\left\{u^{*}\right\}$. By construction, $D$ is a dominating set for $G$. For the edge set of $G^{\prime}$ let $E\left(G^{\prime}\right)=E(G) \backslash E^{*}$, and we further specify that $r=d$. The described instance $I^{\prime}$ of DDS has $k=\left|E^{*}\right|=|V(H)|$. As required, the reduction from $I$ to $I^{\prime}$ can be achieved in polynomial time.

Suppose that $H$ has a dominating set $S$ of size at most $d$. Then in $G^{\prime}$ there must be a dominating set $D^{\prime}=S \cup$ $\left\{u^{*}\right\}$ (of size at most $d+1$ ) and satisfying $d_{\mathrm{v}}\left(D, D^{\prime}\right) \leqslant$ $d=r$. Conversely, suppose that $G^{\prime}$ has a dominating set $D^{\prime}$ and $d_{\mathrm{v}}\left(D, D^{\prime}\right) \leqslant r$. Since $G^{\prime}$ was obtained from $G$ by deleting all of the $k$ edges incident to $u^{*}$ then $S=D^{\prime} \backslash\left\{u^{*}\right\}$ is a dominating set for the subgraph $G^{\prime} \backslash\left\{u^{*}\right\} \equiv H$. Also, $\left|D^{\prime}\right|=d_{\mathrm{v}}\left(D, D^{\prime}\right) \leqslant r=d$ hence $H$ has a dominating set of size at most $d$.

Lemma 5 The unparameterized version of DoMA-VC is NP-complete.

Proof Observe that Dom-A-VC is in NP. Relying on Lemma 4, we reduce from DDS to an instance of Dom-A-VC.

Let $I$ be an instance of DDS. Reduction of $I=$ $(G, G, D, k, r)$ to an instance $I^{\prime}$ of DoM-A-VC follows the process which is described and justified in the proof of Lemma 2. Given such a reduction $I^{\prime}$ of Dom-A-VC, the final part of the proof of Lemma 2 shows that $I$ is a yes-instance of DDS if and only if $I^{\prime}$ is a YES-instance of DOM-A-VC.

Our next objective is to PPT reduce from a suitable parameterized problem to DDS.

\section{ReD-Blue Dominating Set (RBDS)}

Input: A graph $G=(V, E)$ with vertices partitioned $V=R \cup B$, where $R$ and $B$ are independent sets, a positive integer $d$.

Parameter: $(|R|, d)$.

Question: Does there exist $D \subseteq B$ such that $|D| \leqslant d$, and each vertex in $R$ has at least one neighbor in $D$ ?

Dom et al. ${ }^{[29]}$ proved that ReD-BLuE Dominating SET has no polynomial kernel unless 
$\mathrm{NP} \subseteq$ coNP / Poly. The unparameterized version of Red-Blue Dominating Set is known to be NP-hard.

Lemma 6 RBDS has a PPT reduction to DoM-AVC. Thus DOM-A-VC has no polynomial kernel unless NP $\subseteq$ co-NP / Poly.

Proof The proof will follow by a direct application of Corollary 1. We proved in Lemma 5 that the unparameterized version of DoM-A-VC is NP-complete. Given that RBDS has no polynomial kernel $^{[29]}$, and its unparameterized version is NPcomplete, a PPT reduction to DoM-A-VC is sufficient to complete the proof.

Given an instance of RBDS, $I=((R \cup B, E), d)$ where $R$ and $B$ are independent sets, we assume that $R$ has no isolated vertex as otherwise it is trivially a NO-instance. We make the following, trivial, PPT reduction to an instance $I^{\prime}=(G, C, k, r)$ of DomA-VC. Using the same graph, let $G=(R \cup B, E)$, $C=R$, and $r=d$. The size of $C$ is $k=|R|$. Note that $R$ is indeed a vertex cover because $B$ is an independent set. As required, the parameters $k$ and $r$ are polynomial functions, respectively, of $|R|$ and of $d$. Satisfaction of the polynomial time constraint for this reduction is also obvious.

Suppose that $I$ is a yes-instance of RBDS. Then there is a set of at most $d$ vertices, $D \subseteq B$, such that each vertex in $R$ has at least one neighbor in $D$. Then $D$ satisfies the identical condition in $I^{\prime}$, hence $I^{\prime}$ is a yes-instance.

Conversely, suppose that $I^{\prime}$ is a yes-instance of DoM-A-VC. Then $G$ has a set $D$ of at most $r$ vertices such that every vertex in $R$ either belongs in $D$, or has at least one neighbor in $D$. We require slightly more, that $D \subseteq B$. Suppose that there is a vertex $v$ in $R \cap D$. Then $v$ has a neighbor $u$ in $B$, since $R$ is a vertex cover with no isolated vertex. Exchanging each such vertex $v$ in $R \cap D$ with a neighbor $u$ in $B$, we obtain the desired set $D^{\prime}$ of size at most $r=d$. Therefore $I$ is a yes-instance of RBDS.

Theorem 7 The problem DDS has no polynomial kernel unless NP $\subseteq$ co-NP / Poly.

Proof We show that DOM-A-VC has a PPT reduction to DDS.

Let $I=(H, C, k, r)$ be an instance of DoMA-VC. Then $B=V(G) \backslash C$ is an independent set. Similar to the proof of Lemma 4, we construct $G$ from $H$ by adjoining two new vertices $b^{*}, c^{*}$ to $H$, and then adding edges so that $b^{*}$ is adjacent to each vertex in $B$, and $c^{*}$ is adjacent to those in $C$. That is, $V(G)=V(H) \cup\left\{b^{*}, c^{*}\right\}$ and $E(G)=E(H) \cup E^{*}$, where $E^{*}=\left\{\left(v, b^{*}\right) \mid v \in B, v \neq b^{*}\right\} \cup\left\{\left(v, c^{*}\right) \mid v \in C\right.$, $\left.v \neq c^{*}\right\}$. Set $D=\left\{b^{*}, c^{*}\right\}$ is then a dominating set for $G$. Let $G^{\prime}=\left(V(G), E(G) \backslash E_{C}^{*}\right)$ where $E_{C}^{*}=\left\{\left(v, c^{*}\right) \mid v \in C, v \neq c^{*}\right\} \subset E^{*}$. Thus the edge operations taking $G$ to $G^{\prime}$ remove the $k$ edges between $D$ and $C$, hence $C$ has now $k$ non-dominated vertices. This instance $I^{\prime}=\left(G, G^{\prime}, D, k, r\right)$ satisfies the polynomial parameter and time requirements of a PPT reduction. The question concerning $I^{\prime}$ now amounts to the existence, or otherwise, of at most $r$ vertices which dominate the vertices in $C$.

Assume that $I$ is a yes-instance. Then the vertex cover $C$ of size $k$ in $H$ is dominated by a set $D$ of at most $r$ vertices. Then in $G^{\prime}$, there is a dominating set $D^{\prime}$ that contains at most $r+2$ vertices, hence $d_{\mathrm{v}}\left(D, D^{\prime}\right) \leqslant r$. Therefore $I^{\prime}$ is a yes-instance.

Conversely, suppose that $I^{\prime}$ is a yes-instance. Then $d_{\mathrm{v}}\left(D, D^{\prime}\right) \leqslant r$ implies that at most $r$ vertices of $D^{\prime} \backslash$ $D=D$ are needed to dominate $C$. Therefore in $I$, the same vertices in $D$ will dominate $C$. Thus $I$ is a yesinstance.

We have now shown that DoM-A-VC has a PPT reduction to DDS. Collecting this result with Lemmas 4 and 6 , the theorem is proved by direct application of Corollary 1 .

Summarizing this section and the previous one, we have shown our first main result: that the DYNAMIC Dominating SET problem is FPT. Pursuing this positive result a little further, we have shown that although it is FPT, it probably does not have a polynomial kernelization. We next turn to our second storyline.

\section{Turbo-Charging a Greedy Heuristic}

In this section, we prove our second main result: that GREEDY-DS is FPT.

\section{Theorem 8 GREEDY-DS is FPT.}

Proof We reduce the problem to DDS. Given an instance $J=\left(H, L, v, k, D=D_{1} \cup D_{2}\right)$ of GREEDYDS, we can assume that $\left|D_{2}\right| \geqslant 2$ else $J$ is trivial to decide. We can similarly assume that $\left|D_{1}\right| \geqslant 1$, and can choose a vertex $x \in D_{1}$. We create the following instance of DDS.

- $G$ is the graph consisting of the subgraph $H^{\prime}$ of $H$ induced by the set of vertices $U=\left\{v_{1}, \cdots, v\right\} \cup$ $D$, augmented with any edges $x u$ that are not already present in $H^{\prime}$, for all $u \in U$. 
- The evolved graph $G^{\prime}=H^{\prime}$.

- The dominating set $D$ of $G$ is $D_{1}$.

- The edge-edit distance from $G$ to $G^{\prime}$ is the number $k^{*}$ of augmenting edges. (Note that $k^{*} \leqslant k$.)

- The targeted vertex-edit distance to repair $D$ is $r=$ $\left|D_{2}\right|-1$.

Verification of the soundness of the reduction is straightforward.

Discussion. The two storylines, as reflected in our two main results, appear to be closely related. We first found the result that DDS is FPT, and then, inspired by the pioneering work of Hartung and Niedermeier ${ }^{[22]}$ (who proved an FPT result for INCREMENTAL LIST COLORING seemingly motivated by the potential application to FPT-turbo-charging a common greedy heuristic for GRAPH COLORING), we then began to look for a greedy heuristic for DominATING SET for which our FPT result about DDS could serve as the turbo-charging FPT subroutine, thus in some sense, working backwards to the flow of motivation in Ref. [22].

\section{Summary and Research Horizons}

We offer here somewhat surprising FPT results about two parameterizations of the famously $W$ [2]-complete and arguably elemental Dominating SET problem, with two completely different and seemingly quite general, natural storylines as motivation.

The main research horizons, as we see it, are two:

(1) Investigate the parameterized complexity of DYNAMIC $X$ for other $W[1]$-hard problems $X$.

(2) Investigate the parameterized complexity of the naturally defined parameterized problems that arise in attempting to sharpen greedy heuristics, as exemplified by the work of Hartung and Niedermeier (and our Theorem 2, inspired by them).

We remark that with respect to the research program of sharpening greedy heuristics, there is nothing to lose! If one sets the back-up parameter at $k=0$, then one simply has the original greedy routine currently in use. Beyond "nothing to lose" we believe that both research directions may be quite practical and profitable - but to determine if this is so will require some serious engagement with algorithms engineering and implementation, such as in Ref. [22].

Lastly, whether the FPT "turbo-charged" greedy heuristic for Minimum Dominating SET has practical value is as yet undetermined, and is the subject of a sequel investigation. Preliminary results seem to be encouraging.

\section{Acknowledgements}

This research was partially supported by the Australian Research Council.

\section{References}

[1] R. M. Karp, Heuristic algorithms in computational molecular biology, J. Comput. Syst. Sci., vol. 77, no. 1, pp. 122-128, 2011.

[2] L. Cai, J. Chen, R. G. Downey, and M. R. Fellows, On the structure of parameterized problems in NP, Inf. Comput., vol. 123, no. 1, pp. 38-49, 1995.

[3] L. Cai, J. Chen, R. G. Downey, and M. R. Fellows, Advice classes of parameterized tractability, Ann. Pure Appl. Logic, vol. 84, no. 1, pp. 119-138, 1997.

[4] L. Cai, J. Chen, R. G. Downey, and M. R. Fellows, On the parameterized complexity of short computation and factorization, Arch. Math. Log., vol. 36, nos. 4-5, pp. 321337, 1997.

[5] J. Chen, Randomized disposal of unknowns and implicitly enforced bounds on parameters, in IWPEC, 2008, pp. 1-8.

[6] J. Chen, Vertex cover kernelization, in Encyclopedia of Algorithms, M. Y. Kao, ed. Springer, 2008.

[7] J. Chen, Maximum partition matching, in Encyclopedia of Opti-Mization. Kluwer Academic Publishers, 2009, pp. 2029-2035.

[8] J. Chen and S. B. Cooper, Algorithms, complexity and computational models, Theor. Comput. Sci., vol. 412, no. 23, pp. 2457-2458, 2011.

[9] J. Chen, H. Fernau, I. A. Kanj, and G. Xia, Parametric duality and kernelization: Lower bounds and upper bounds on kernel size, in STACS, 2005, pp. 269-280.

[10] J. Chen and I. A. Kanj, Parameterized complexity and subexponential-time computability, in The Multivariate Algorithmic Revolution and Beyond, 2012, pp. 162-195.

[11] J. Wang, Q. Feng, and J. Chen, Color-coding and its applications: A survey, Int. J. Software and Informatics, vol. 5, no. 4, pp. 595-606, 2011.

[12] R. G. Downey and M. R. Fellows, Parameterized complexity, in Monographs in Computer Science. SpringerVerlag, New York, 1999.

[13] R. G. Downey and M. R. Fellows, Fundamentals of Parameterized Complexity. Texts in Computer Science. Springer-Verlag, London, 2013.

[14] J. Flum and M. Grohe, Parameterized Complexity Theory. Springer, 2006.

[15] R. Neidermeier, Invitation to Fixed-Parameter Problems, Oxford Lecture Series in Mathematics and its Applications. Oxford University Press, 2006.

[16] H.-J. Böckenhauer, J. Hromkovic, and A. Sprock, On the hardness of reoptimization with multiple given solutions, Fundam. Inform., vol. 110, nos. 1-4, pp. 59-76, 2011.

[17] H.-J. Böckenhauer, J. Hromkovic, T. Mömke, and P. Widmayer, On the hardness of reoptimization, in SOFSEM, 
volume 4910 of LNCS, Springer, 2008, pp. 50-65.

[18] U. Stege. Private communication, 1998.

[19] R. Görke, P. Maillard, C. Staudt, and D. Wagner, Modularity-driven clustering of dynamic graphs, in SEA, 2010, pp. 436-448.

[20] S. Basu, I. Davidson, and K. Wagstaff, Constrained Clustering: Advances in Algorithms, Theory, and Applications. Chapman and Hall, 2008.

[21] H. Shachnai, G. Tamir, and T. Tamir, Minimal cost reconfiguration of data placement in a storage area network, Theor. Comput. Sci., vol. 460, pp. 42-53, 2012.

[22] S. Hartung and R. Niedermeier, Incremental list coloring of graphs, parameterized by conservation, Theoretical Computer Science, 2013.

[23] R. G. Downey, M. R. Fellows, and U. Stege, Parameterized complexity: A framework for systematically confronting computational intractability, in DIMACS Series in Discrete Mathematics and Theoretical Computer Science, 1999, pp. 49-99.

[24] H. L. Bodlaender, R. G. Downey, M. R. Fellows, and D. Hermelin, On problems without polynomial kernels, Journal of Computer and System Sciences, vol. 75, no. 8,

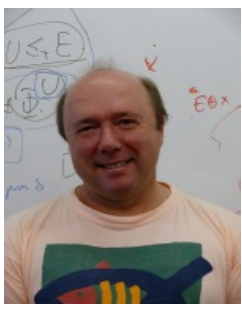

Rod Downey received his $\mathrm{PhD}$ degree in 1982 from Monash University, and holds a chair at Victoria University, New Zealand. He works in the theory of computation, computational complexity and algorithmic randomness. His prizes include the inaugural MacLaurin Fellowship, the Hamilton Prize, and Hector Medal of the Royal Society of NZ, the New Zealand Association of Scientists Research Medal, the New Zealand Mathematics Society Research Award, and EATCS Nerode Prize, and the ASL Shoenfield Prize in Logic. In 2009, he was a James Cook Fellow and in 2012 a fellow at the Newton Institute in Cambridge. He was elected a fellow the Royal Society of New Zealand, the New Zealand Mathematics Society, the American Mathematics Society, the Australian Mathemathics Society, and the Association for Computing Machinery. He has three books including two with Mike Fellows, Parameterized Complexity (1999) and Fundamentals of Parameterized Complexity (2014).

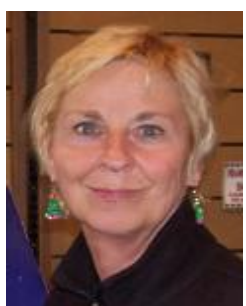

Frances A. Rosamond received her $\mathrm{PhD}$ degree from Cornell University, Ithaca, NY. Her research area in parameterized complexity focuses on kernelization and computational social choice. Rosamond is Editor of the Parameterized Complexity Newsletter, and Moderator of the Parameterized Complexity wiki located at http://FPT.wikidot.com. Professor Rosamond is active in mathematical sciences popularization (www.CSMaths.org), and offers workshops using activities from the program, "Computer Science Unplugged!" Frances Rosamond is a research professor at Charles Darwin University, Northern Territory, Australia. pp. 423-434, 2009.

[25] H. L. Bodlaender, S. Thomass'e, and S. Yeo, Kernel bounds for disjoint cycles and disjoint paths, Theoretical Computer Science, vol. 412, no. 35, pp. 4570-4578, 2011.

[26] L. Fortnow and R. Santhanam, Infeasibility of instance compression and succinct PCPs for NP, in 40th Annual International Symposium on Theory of Computing (STOC 08), 2008, pp. 133-142.

[27] M. Cygan, F. V. Fomin, L. Kowalik, D. Lokshtanov, D. Marx, M. Pilipczuk, M. Pilipczuk, and S. Saurabh, Parameterized Complexity. Springer. To appear in 2014 December.

[28] M. R. Garey and D. S. Johnson, Computers and Intractiblity. A Guide to the Theory of NPCompleteness. W. H. Freeman, New York, 1979.

[29] M. Dom, D. Lokshtanov, and S. Saurabh, Incompressibility through colors and IDs, in Automata, Languages and Programming. Springer, 2009, pp. 378-389.

[30] D. Binkele-Raible, H. Fernau, F. V. Fomin, D. Lokshtanov, S. Saurabh, and Y. Villanger, Kernel(s) for problems with no kernel: On out-trees with many leaves, ACM Trans. Algorithms, vol. 8, no. 4, pp. 38:1-38:19, 2012.

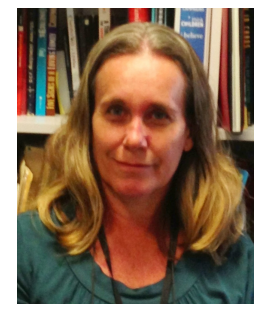

Judith Egan received her PhD degree from Monash University, Australia, in 2010 for her thesis on Transversals, Indivisible Plexes and Partitions of Latin squares. Her recent research includes topics in parameterized complexity, combinatorial mathematics, algorithm engineering, and programming for high-performance computing. Judith is a University Fellow at Charles Darwin University (CDU) and also teaches mathematics at CDU and in high schools.

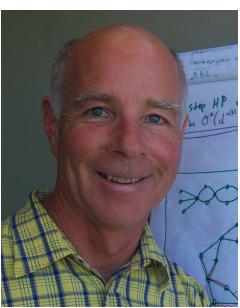

Michael R. Fellows (Charles Darwin Univ., Australia), received his $\mathrm{PhD}$ degree in computer science from the Univ. California, San Diego (his hometown) in 1985. He is well-known for his foundational work in parameterized complexity (Springer Festschrift LNCS 7370) and has received numerous awards, including an Alexander von Humboldt Research Prize (in 2006) and the Nerode Prize (in 2014). In 2014, he was honored as one of the ten inaugural Fellows of the EATCS.

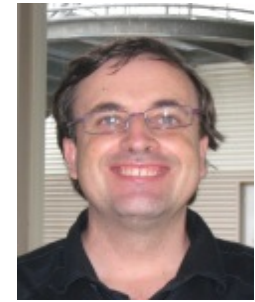

Peter Shaw received his $\mathrm{PhD}$ degree from The University of Newcastle, Australia in 2010. His research areas are currently in the application of parameterized complexity in the analysis of big data sets and using parameterized complexity to improve heuristics. Peter Shaw is currently a Senior Lecturer at Charles Darwin University, Northern Territory, Australia. 